\title{
Dr. Ricardo Bustamante Bozzo Maestro de la Anestesiología Chilena
}

Durante la celebración del XXIV Congreso Chileno de Anestesiología en Termas de Chillán en el año 1996 y luego de haber hecho la presentación del Dr. Enrique García Müller como Maestro de la Anestesiología Chilena en un acto similar a este, el Dr. Hector Lacassie Silva regresó a su asiento en la primera fila de la audiencia, ubicándose delante de donde se encontraba sentado el Dr. Ricardo Bustamante y yo.

El Dr. Ricardo Bustamante le dijo al Dr. Lacassie "Amigo, ojalá que en algún momento yo tenga la oportunidad de presentarte a ti para la misma distinción”. Probablemente ambos lo recuerden.

Lo que yo nunca le he contado a mi amigo Ricardo hasta este momento, es que al escuchar eso, dos ideas cruzaron por mi mente. La primera fue "Ojalá que en algún momento yo te pueda otorgar esa oportunidad", la segunda "Ojalá que yo también tenga la oportunidad de presentarte a ti para la misma distinción".

El año 2010, durante la celebración del XXXVIII Congreso Chileno de Anestesia en la ciudad de Puerto Varas, un emocionado Ricardo Bustamante presentaba a su amigo Héctor Lacassie Silva como Maestro de la Anestesiología Chilena. Yo había contribuido a la nominación de Ricardo como presentador del Dr. Héctor Lacassie en mi calidad de Presidente de la Sociedad de Anestesiología de Chile y con ello, había logrado que Ricardo cumpliera su deseo y yo el primero de los míos.

Hoy, cierro un ciclo que duró 19 años desde ese congreso en 1996, al tener la oportunidad de cumplir con el segundo de mis anhelos: presentar al Dr. Ricardo Bustamante como Maestro de la Anestesiología Chilena. Es por ello que en primer lugar debo agradecer al Directorio de la Sociedad de Anestesiología de Chile el haberme distinguido con este honor.

\section{Ricardo el Ser Humano}

Hijo de Don Bertín Bustamante Espinoza y

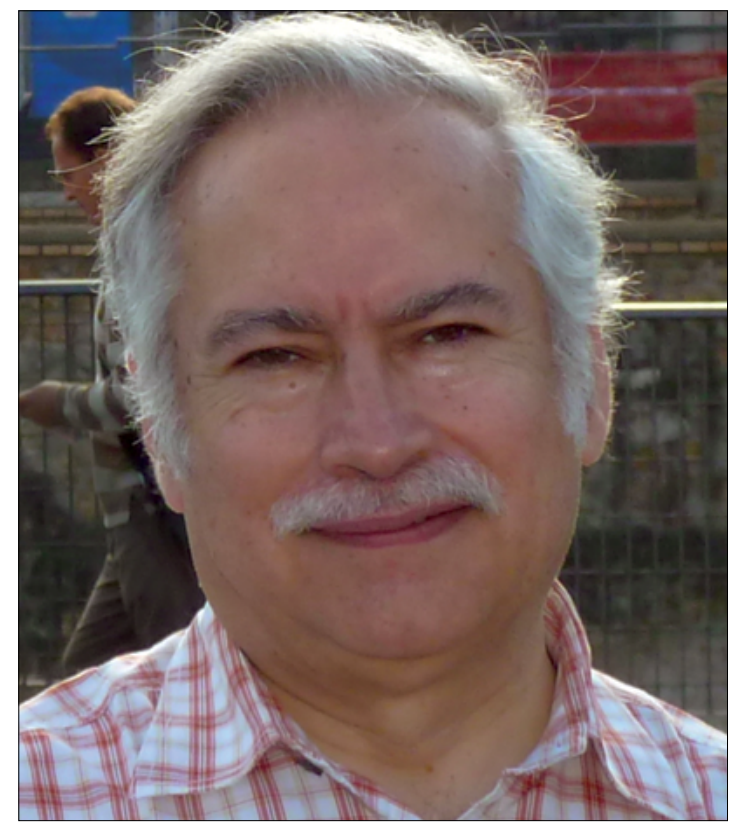

Dr. Ricardo Bustamante.

Doña Amadea Bozzo Delledonne. Don Bertín, médico cirujano y profesor de urología de la Universidad de Chile y Doña Amadea, químico farmacéutica y una de las primeras mujeres en obtener un título universitario en Chile... "lo que se hereda, no se hurta".

Miembro de una familia de 4 hermanos: Teresa, Fernando, Bertín y Ricardo, constituyó luego su propia familia junto a su esposa Marisol, sus hijos Felipe y Alejandra y hace muy poquito tiempo ésta se completó con la llegada de sus nietos Vicente y Nicolás.

Si tuviera que definirlo con dos conceptos que lo distinguen elegiría: melómano y neurótico obsesivo... y ambos conceptos muy bien entrelazados uno con el otro.

La melomanía nos ha unido por mucho tiempo, claro que él es peor. Ha pasado por el vinilo, el disco láser, el CD, el DVD, el Blueray y el IPod... No sé si llegó a Spotify, supongo que no, 


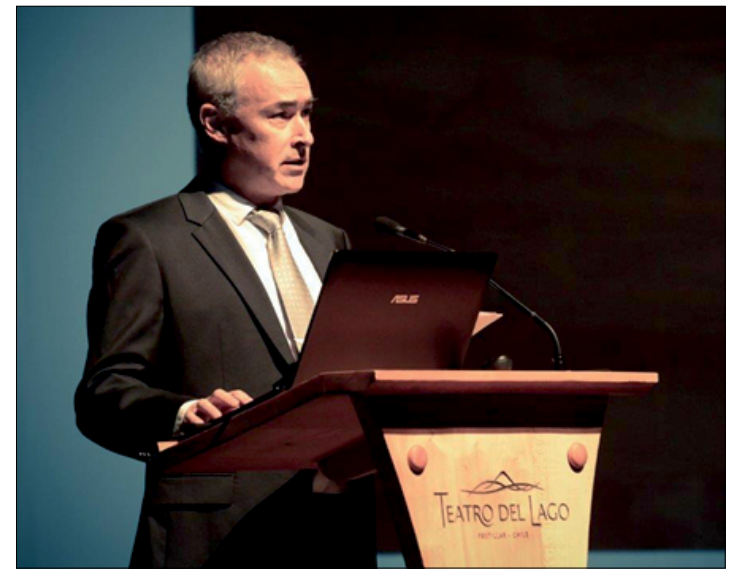

no le gusta que la pauteen.

- Más de 4.000 CDs en una pieza especialmente acondicionada para ordenarlos.

- Cada caja con un numerito en el lomo que se encuentra registrado en un libro, donde aparece el detalle de ese disco.

- En el reverso de la tapa de cada CD, un papelito amarillo autoadhesivo con unos números manuscritos. Esos son los temas que le gustan de ese CD.

Por años me he entretenido diciéndole: "Te apuesto que no tienes nada de ---------", seguido por el nombre de un artista, ojalá lo más rebuscado posible, viendo cómo acto seguido, saltaba de su asiento entusiasmado y orgulloso y me traía no uno sino tres discos de ese artista.

En una oportunidad le dije: "Te apuesto que no tienes nada de la Ramona Galarza", a lo que en su tono soez habitual me respondió: "Me $\mathrm{Ca}$ gaste".

Una semana después en la Feria del Disco, perdido entre los CDs de tango, encontré un disco de Ramona Galarza. Inmediatamente pensé: "Me lo cagué".

A la semana siguiente me encontré con Ricardo y le dije: "Mira lo que tengo", a lo que él me respondió "Ah si, yo tengo dos".

$\mathrm{Su}$ Ipod repleto de 160 gigas de música de todos los tipos. Desde Chico Trujillo a Brahms, pasando por sus intermediarios naturales que son Buddy Richard, Los Beatles y Elton John.

¿Ven a lo que me refiero con melómano y neurótico obsesivo?

Esa misma neurosis obsesiva la ha llevado al

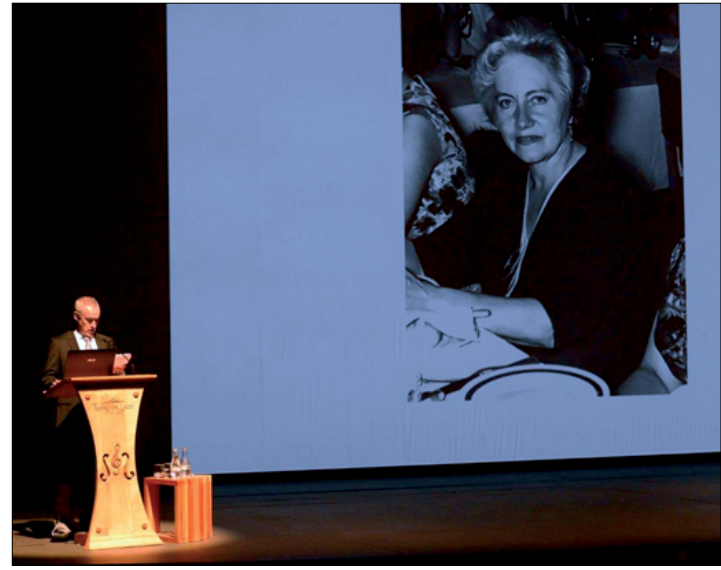

ámbito profesional como anestesista, realizando investigación, escribiendo artículos y capítulos de libros, como Secretario de la Sociedad de Anestesiología de Chile, como Editor de la Revista Chilena de Anestesia y en cualquier tarea que se le haya encomendado. Su trabajo es siempre garantía de excelencia y rigurosidad.

\section{Ricardo el Estudiante y el Profesional}

Brillante, meticuloso, nuevamente obsesivo.

Realizó sus estudios primarios y secundarios en el Instituto Alonso de Ercilla.

Estudió Medicina en la Universidad de Chile, entre los años 1969 y 1976, obteniendo el Premio Colegio Médico de Chile al mejor egresado de la Escuela de Medicina de la Universidad de Chile, promoción 1976.

El mismo año 1976 inició su postgrado en Anestesiología en la Pontificia Universidad Católica de Chile, siendo el integrante único de dicha promoción y egresando el año 1979.

Una vez egresado de su beca, trabajó en la Universidad Católica por dos años, luego de lo cual se contrató en el Servicio de Anestesiología de la Posta Central (actual Hospital de Urgencia Asistencia Pública), donde aún permanece, completando 34 años de vida laboral. Quizás si uno de sus mayores méritos: pese a haber tenido la oportunidad de trabajar en cualquier lugar y con condiciones por cierto muchos mejores, haber permanecido fiel a su querida Posta Central, siendo consecuente con sus creencias políticas y humanas. Entregando su mejor versión para los 
pacientes más pobres del País.

Fue en la Posta Central, donde en conjunto con los Drs. Carlos Reyes y Víctor Hanna participó en la creación de una beca no universitaria, muy resistida por muchos inicialmente, muy resistida por menos posteriormente, que luego se convirtió en el Programa Ministerial de Formación de Anestesistas y a partir del año 1999 en el Programa de Postgrado en Anestesiología de la Universidad de Santiago de Chile.

A diciembre de 2014, dicho programa le había aportado al país 143 anestesiólogos, ocupando el tercer lugar luego de la Universidad de Chile con 280 anestesiólogos y la Pontificia Universidad Católica de Chile con 270 anestesiólogos.

Lo que no cabe duda es que, independiente del rol más o menos activo que ha jugado en las distintas etapas de su desarrollo, la presencia del Dr. Ricardo Bustamante en este programa, ha significado un elemento de prestigio y una suerte de garantía de seriedad para muchos, dentro de la comunidad anestésica.

Sin embargo, este factor no debe soslayar el aporte docente efectivo que ha hecho a la formación de este centenar y medio de especialistas. Yo como muchos, recuerdo cuando fui asignado al quinto turno en nuestra beca, el turno del Dr. Ricardo Bustamante.

No me cabe duda que todos quienes por ahí transitamos, recordamos la recepción cálida, las reglas claras, las llamadas de atención, los conocimientos infinitos, el apoyo constante, la confianza progresiva, el sentirse respaldado.

Es por eso que te pido Ricardo que cuando subas al estrado, en algún momento entre tus palabras probablemente tratando de argumentar lo inmerecido que consideras esta distinción, hurgues entre la asistencia y vas a encontrar muchos de esos 150 rostros que te observarán con un gesto de gratitud. Luego puedes continuar con tu discurso, pero estoy seguro que en ese momento habrás percibido parte de lo que tú has hecho por la anestesiología del País.

\section{Ricardo el Maestro}

Quiero empezar esta parte de mi presentación mostrando pruebas gráficas en que aparezco junto con Ricardo en Venecia, en Atenas y en el Santiago Bernabéu. Faltan muchos lugares que

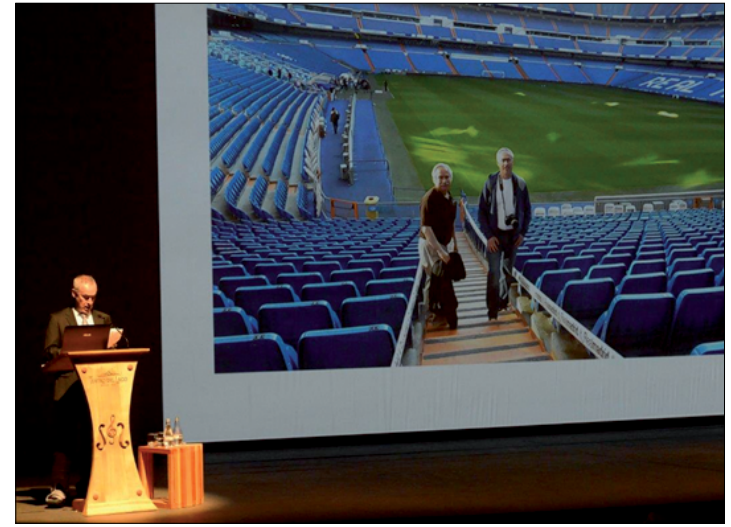

no incluyo para no aburrirlos.

Con ello quiero declarar ante esta audiencia que Ricardo es mi entrañable amigo y que en consecuencia, mi presentación no es imparcial... Como no lo fue Guillermo Lema al presentar al Dr. Jorge Urzúa, ni Héctor Lacassie Silva al Dr. Enrique García Müller, ni el propio Ricardo al Dr. Héctor Lacassie, ni Mario Concha al Dr. Samuel Torregrosa, ni Ignacio Cortínez al Dr. Hernán Muñoz, ni Luis Brunet a la Dra. Lucía Volosky, ni Héctor Lacassie Quiroga al Dr. José de la Fuente... Tampoco Mario Concha al Dr. Jorge Dagnino.

Todas ellas fueron presentaciones en que se encomendó a un discípulo y además a un amigo, el mostrarle a la audiencia las características, los afanes y las virtudes de alguien, que para ellos se constituía en un ejemplo de lo que debe ser un Maestro.

Mi responsabilidad es la misma, desde lo profundo de mi sesgo y en consecuencia, voy a comenzar por mencionar someramente los elementos menos sesgados en este análisis, que son los requisitos que la Sociedad de Anestesiología impone para ostentar esta distinción, que surgieron del trabajo de una comisión conformada en el período presidencial del Dr. Alejandro González y que entre otros, contó con la participación de los Drs. Jorge Dagnino y Ricardo Bustamante.

Dichos requisitos incluyen: Edad mayor de 65 años (no sé porqué); permanencia en la Sociedad por más de 25 años; trabajo gremial o científico en la sociedad por un período mayor de 10 años como miembro del directorio, miembro del comité editorial de la Revista Chilena de Anestesia, comités de subespecialidades, organización 
o participación en congresos y otros; que posea publicaciones y finalmente y en el más subjetivo de los requisitos: que exista consenso en el Directorio, que ha participado en forma decisiva en la formación de anestesiólogos y en el desarrollo de la especialidad.

Además, de los años de vida y los años de permanencia en la Sociedad de Anestesiología, sus dos años de secretario del Directorio entre los años 1991 y 1993, sus trece años de participación en el Comité Editorial de la Revista Chilena de Anestesia, sus siete años como Editor Principal de la Revista Chilena de Anestesia, sus múltiples participaciones como conferencista y panelista en el Congreso Chileno de Anestesia, incluyendo una Conferencia Plenaria Profesor Cabrera, sus múltiples capítulos de libros y revistas científicas relacionados con su tema predilecto (bloqueadores neuromusculares), sus trabajos de investigación dentro de los cuales tres han sido merecedores a la distinción de mejor trabajo del Congreso Chileno de Anestesiología, hacen evidente que el Dr. Ricardo Bustamante cumple con creces con todos los requisitos que he señalado previamente.

Y sin embargo, cabe preguntarse si este nutrido currículum convierte a Ricardo en un Maestro de la Anestesiología. La respuesta es no.

$\mathrm{Y}$ es no porque estos requisitos establecidos como un piso mínimo, no son más que eso, un piso mínimo para definir el "desde", aquellos atributos basales sobre los cuales se debe empezar a deliberar y que incluso en algunas ocasiones se deben obviar. Es el caso del Dr. Hernán Muñoz, quien no cumplía con varias de estas características y sin embargo, nadie duda que era merecedor de la distinción de Maestro de la Anestesiología Chilena.

Por otro lado, hay quienes pudieran pensar que ese otro requisito subjetivo "consenso en que ha participado en forma decisiva en la formación de anestesiólogos y en el desarrollo de la especialidad", es el elemento más importante a tener en consideración.

Probablemente lo es, sin embargo, nuevamente, ¿El haber formado anestesiólogos en forma decisiva enviste a alguien del título de Maestro?... Nuevamente la respuesta es no. Y es no especialmente ahora que los programas de formación de anestesistas en Chile ya no son cinco, sino dieciséis.
En este aspecto, difiero de lo señalado por el propio Ricardo en un Editorial de la Revista Chilena de Anestesia, en que augura que al haber más programas de postgrado y al ser más los anestesistas del país, es esperable un aumento de la cantidad de Maestros de la Anestesiología.

Las generaciones han cambiado, los compromisos son más efímeros, las relaciones con las instituciones son más débiles. No les pido que me respondan, pero le pregunto a todos aquellos asistentes a esta ceremonia que pertenecen a las nuevas generaciones de anestesiólogos o becados ¿Cuántos de ustedes se imaginan trabajando en la Posta Central por 34 años?

En mi opinión, la distinción de Maestro de la Anestesiología Chilena va a continuar siendo merecida por un grupo reducido de especialistas, puede ser que un poco más nutrido, pero aún así reducido a aquellos que conviertan sus años de trabajo en un continuo de enseñanza y formación profesional para las nuevas generaciones que nos sucedan.

En consecuencia, la definición de Maestro pasa inevitablemente por un análisis estrictamente subjetivo, que no puede ser incorporado en los requisitos y que evidentemente queda encomendado a aquellos que han sido los discípulos. Puede que la Sociedad de Anestesiología se equivoque una o muchas veces, en la nominación o en la no nominación de alguien para este honor, pero todos nosotros sabremos, obtenga o no el galardón, a quien o a quienes consideraremos nuestro o nuestros maestros.

Y como yo me encuentro en esa posición respecto a la figura del Dr. Ricardo Bustamante, me voy a permitir en estos últimos minutos describir dos de sus atributos que representan para mí la esencia de lo que significa un Maestro.

Primero, el Dr. Ricardo Bustamante no es un líder. Si yo lo viera cavando un hoyo no tomaría una pala ni me pondría a cavar a su lado. Lo más probable es que sólo encontráramos tierra. Sin embargo, si yo decidiera cavar mi propio hoyo, le pediría que me acompañara, que tomara una pala y que me ayudara a cavar y cada cierto tiempo, detendría mi trabajo, dejaría la pala al lado y le preguntaría ¿Cómo crees que lo estamos haciendo? ¿Crees tú que estamos cavando bien? Y su respuesta influiría decisivamente en como continuáramos el trabajo.

La maestría tiene muchas veces el mérito de 
influir desde bambalinas en cómo se ejecuta la obra.

Segundo, cuando uno se pregunta porqué el color de los cinturones en las artes marciales va oscureciendo progresivamente en la medida que el alumno avanza en sus habilidades y destrezas, se encuentra con el siguiente párrafo:

El centro del sol es blanco... Al expandir su luz hacia el infinito el rayo de sol se observa amarillo... y luego naranja ... después azul... a medida que alumbra más lejos se torna verde... morado... café... Hasta llegar finalmente al negro en donde se confunde con la oscuridad de la noche...

Un cinturón negro se interna en la oscuridad de las tinieblas para dar la batalla contra el monstruo de las mil cabezas...

Si logra triunfar esta batalla nacerá en un nuevo día donde un blanco sol le recibirá alumbrando su afilada Katana espiritual.

Su cinturón negro ha emblanquecido, ahora por las mil batallas que el valiente guerrero libró lleno de pureza y dignidad en su corazón por alcanzar los nobles ideales señalados tiempo atrás por su sabio maestro...

Estas palabras llenas de metáforas, contienen una serie de códigos que vale la pena descifrar.

El cinturón negro es el final de un camino y el inicio de otro. En ese otro camino el guerrero se internará en las tinieblas y peleará contra el monstruo de las mil cabezas que no es otra cosa que su propio ego. Si en esas tinieblas el guerrero logra no ser devorado por su principal enemigo que es él mismo, logrará acceder a la luz. En ese momento su cinturón se habrá convertido nuevamente en un cinturón blanco.

Todo aquel que practica artes marciales sabe que en la medida que el poseedor de un cinturón negro continúa sus entrenamientos, el desgaste hace que en los bordes de su cinto aparezcan incipientes trazos blancos. La verdadera maestría se logra cuando el cinturón vuelve a ser blanco.

En esta sociedad en que trabajamos en que existen tantos anestesistas recientemente egresados, que lucen orgullosos y ufanos los cinturones negros que los distinguen como expertos en conocimientos y habilidades, es muy reconfortante ver transitar al Dr. Ricardo Bustamante con su cinturón blanco a donde quiera que llegue, dispuesto a enseñar todo lo que sabe y dispuesto a aprender del más novel de sus discípulos.
Ahí está la esencia de su maestría y es por eso por lo que hoy el Dr. Ricardo Bustamante Bozzo debe sentir pleno orgullo de convertirse en uno más de los Maestros de la Anestesiología Chilena.

Muchas Gracias.

\section{Dr. Renato Chacón Abba}

Srs Miembros del Directorio de la Sociedad Chilena de Anestesiología, estimados colegas y amigos:

Recibí hace algunos meses un e-mail de la Sociedad con el asunto "Carta de agradecimiento". Estaba fuera de Santiago y postergué la lectura del archivo pensando que se trataba de agradecer la culminación de 7 años de trabajo como Editor de la Revista Chilena de Anestesia. La verdad sea dicha, fue una tarea ingrata, aceptada después de un prolongado parto de argumentos, bajo una larga y continua peridural de escepticismo de mi parte en relación a mis capacidades para ejercer tal cargo. Finalmente accedí; el trabajo fue duro y me parecía que la carta de agradecimiento podría tener algún fundamento.

Mi desconcierto fue total al leerla posteriormente y enterarme que se trataba de mi designación como Maestro de la Anestesiología Chilena, que es el más importante galardón que la $\mathrm{SACH}$ pueden entregar a sus asociados más destacados. Fue un balde de agua fría de perplejidad. Tuve dos reacciones simultáneas: la primera fue pensar que no había duda que en ese momento había personas que realmente lo merecían y mi primer pensamiento fue hacia Jorge Dagnino; la segunda fue que tenía que renunciar a tal distinción porque no me correspondía. La primera reacción la solucioné preguntando si había otro nombramiento este año, y fue muy tranquilizador saber que Jorge había sido también nominado para tal efecto. La segunda, la repensé y me pareció un grado de soberbia mayor rechazar la nominación, más que un signo de humildad frente a una distinción que me parecía absolutamente inmerecida.

Lo siguiente fue pensar en este momento, al estar frente a Uds, como iba a justificar la barbaridad que había cometido el Directorio y como 
enfrentar el nerviosismo de estar en esta ceremonia para intentar decir algo inteligente. Para tratar de estar a la altura. Lo primero lo intelectualicé pensando que quién iba a tener que justificar lo injustificable era la persona que me presentara; en este caso, mi entrañable amigo el Dr. Renato Chacón, cosa que entonces no sabía. Lo segundo, tendré que sufrirlo de todas maneras a partir de este momento.

Como hice saber al Directorio, probablemente mi mayor orgullo es recibir esta máxima distinción fundamentalmente como un reconocimiento a mi trabajo en el sector público.

\section{Primer intento}

Mi primer intento de discurso fue fallido. Se trataba de no ser extremadamente escueto ni extremadamente extenso en mis palabras, pensando en que a esta hora lo que todos queremos es relajarnos, después de una agotadora sesión de Congreso. Sin embargo, la mitad del tiempo la ocupaba tratando de explicar porqué yo no debía haber sido nominado $\mathrm{y}$, la otra mitad, dando las razones por las cuales mi amigo Jorge sí debía serlo. Tuve que rehacerlo, pero sin dejar de decir algunas palabras en relación a ambos aspectos.

Probablemente lo más intimidante es la palabra "Maestro". Ya en otras oportunidades se ha acudido al diccionario de la RAE para encontrar la acepción más adecuada a esta distinción y, obviamente, a mí no me calza. Un maestro no es sólo la persona que traspasa sus conocimientos a sus discípulos, sino que les deja una marca indeleble para toda la vida. Todos nos acordamos de algún maestro del colegio que fue capaz de traspasar esa frontera de trasmitir sólo conocimientos. En la universidad es más difícil, aunque los hubo. En el postgrado es casi imposible. Por eso hay pocos y deberían ser delicadamente seleccionados.

Un maestro debe ser una persona inteligente capaz darse cuenta de las capacidades específicas de cada uno de sus discípulos; debe ser capaz de intuir cuando, muchas veces, éstos son mejores que ellos mismos y hay que dejarlos volar con alas propias; pero este no es un premio a la inteligencia. Un maestro no debe ser necesariamente un buen administrador, pero sí darse cuenta de que es incapaz de hacerlo bien cuando corres-

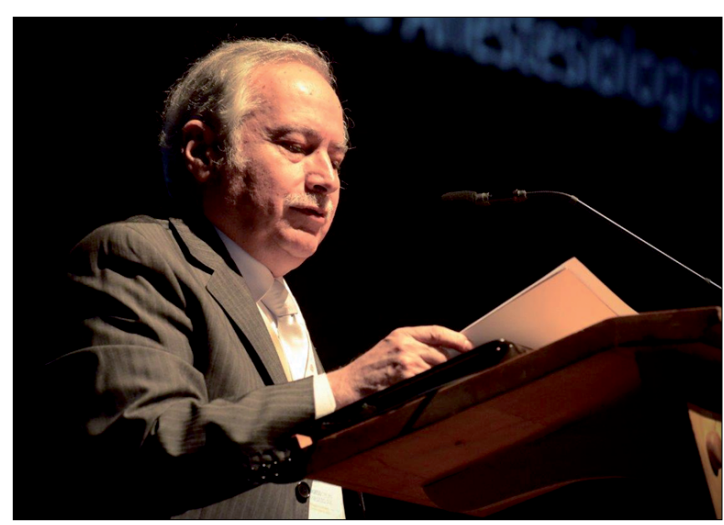

ponde; pero tampoco este es un premio a la gestión.

Esto me trae a la memoria una anécdota del Dr. Mario Folch, que después de haber sido el primer Profesor Titular de la Cátedra de Anestesiología del Hospital Clínico de la Universidad de Chile y haber tenido todos los merecimientos, en 1970 dejó el cargo de Jefe de Departamento al Dr. Felipe Olivarí, diciendo "yo no soy buen administrador, sino un buen técnico" y permaneció como docente hasta 1977, año en que se acogió a jubilación. Ese es un verdadero maestro. Aunque en rigor Don Felipe fue Maestro de la Anestesiología Chilena antes que el Profesor Folch, finalmente se hizo justicia y, ya enfermo, fue nominado en 1995, tres años antes de su muerte.

Trato de explicar que esta distinción debe ser entregada a aquellas personas respetadas por sus pares no sólo por sus conocimientos, por su criterio, por su inteligencia, sino fundamentalmente por la visión universal de todas las disciplinas involucradas en el proceso de educar: la anestesiología no es incompatible con la filosofía, la literatura, las artes plásticas, la música. Además la anestesiología está relacionada de una u otra forma con todas las especialidades médicas y, un maestro, debe tener esa visión.

Todo aquél que me conoce sabe que he tratado de guardar siempre un perfil bajo y pasar inadvertido. Este nombramiento ha hecho exactamente lo contrario, trastocando un poco mi cotidianeidad. Mi único consuelo es que el asunto no estará en el tapete por mucho tiempo. Por esta forma de ser, un poco relacionada con un trastorno de ansiedad social, utilizando distintos argumentos, he eludido las jefaturas, porque me 
he sentido incapaz de ejercerlas. Reconozco que el cargo se me ofreció en un par de oportunidades, pero siempre encontré alguna excusa; mi lema era: "es mejor ser amigo del jefe que ser jefe". Me gustaban aquellos tiempos en que las jefaturas se ganaban por merecimientos y no se ofrecían; cuando llegaron esos tiempos, encontré otras justificaciones.

Estoy muy orgulloso también de saber que esta iniciativa deriva completamente de mi trabajo en la Asistencia Pública. He sido invitado a Cursos de Postgrado y Congresos nacionales e internacionales, con el único aval de haber trabajado en esta institución. Toda la investigación clínica que pude hacer, con mayor o menor éxito, fue realizada en esta institución y con la colaboración de mis colegas del Servicio de Anestesia.

Indudablemente sería antinatural que estuviera disgustado. He recibido verdaderas muestras de alegría y congratulaciones no solo de mis amigos, sino sorprendentemente, de una gran cantidad de personas con las que nunca había tenido mucha cercanía. Estoy muy agradecido a los miembros de este Directorio, que finalmente se limitaron a aplicar las reglas de juego que yo mismo colaboré a elaborar: dos de los miembros de esas comisión están siendo distinguidos hoy. Se produce como consecuencia una inesperada contradicción que deja sólo dos alternativas: o se cambian las reglas de juego, o el número de maestros va a aumentar significativamente porque la vara quedó puesta muy baja.

Sobre los merecimientos de Jorge Dagnino para esta nominación se ha dicho mucho, pero no lo suficiente. Mi gran satisfacción es haber sido su amigo desde que nos conocimos: él becado de tercero, yo becado de primero. No sé porqué dos personaje tan disímiles han podido mantener una gran amistad durante tantos años; el afecto es mutuo. Creo que una de las explicaciones más razonables es que nuestras señoras son también muy amigas. Es casi un silogismo: dos hombres casados no pueden ser amigos si sus mujeres no lo son.

Para mí definitivamente es un honor compartir en el mismo año esta distinción con Jorge Dagnino, aunque en justicia, e incluso de acuerdo a los propios reglamentos, él debió haberlo sido antes que yo. Para que no hubiera dudas al respecto, solicité al Directorio que su ceremonia de nombramiento fuera antes que la mía, para que quedara claro que sus merecimientos eran evidentemente mayores que los míos. Se ha transformado en una tradición que los maestros sean nombrados de a PARES: creo que no es necesario, ni menos en este caso: ¡NONES!

\section{La Escuela de Atenas}

Quiero hacer algunos recuerdos sobre mi llegada a la beca de anestesiología al Servicio de Anestesiología de la Universidad Católica (en esa época no se usaba el PUC, ni menos el Christus). Llegué como egresado de la Universidad de Chile, sin conocer a nadie y, sin embargo, fui tratado con gran afecto por los docentes que me iniciaron en el camino de la anestesiología.

Mario Allende era el Jefe de Servicio (aún no existía el Departamento de Anestesia, ni menos la División). Pensando a posteriori, estaba inmerso en La Escuela de Atenas: habitualmente en el antiguo pabellón 6 estaba el Dr. Waldemar Badía (futuro Maestro de la Anestesiología Chilena); compartiendo conmigo los pabellones del tercer piso, como uno más de ellos y, compartiendo también una gran y perdurable amistad estaban José de la Fuente y Samuel Torregrosa (futuros Maestros de la Anestesiología Chilena); en el sexto piso, haciendo anestesia cardiovascular, estaba Jorge Urzúa (también futuro Maestro de la Anestesiología Chilena); finalmente, en el primer piso, haciendo anestesia obstétrica, estaba Héctor Lacassie (a quien yo tuve el honor de presentar como Maestro de la Anestesiología Chilena). Jorge Dagnino volvía ya en tercer año

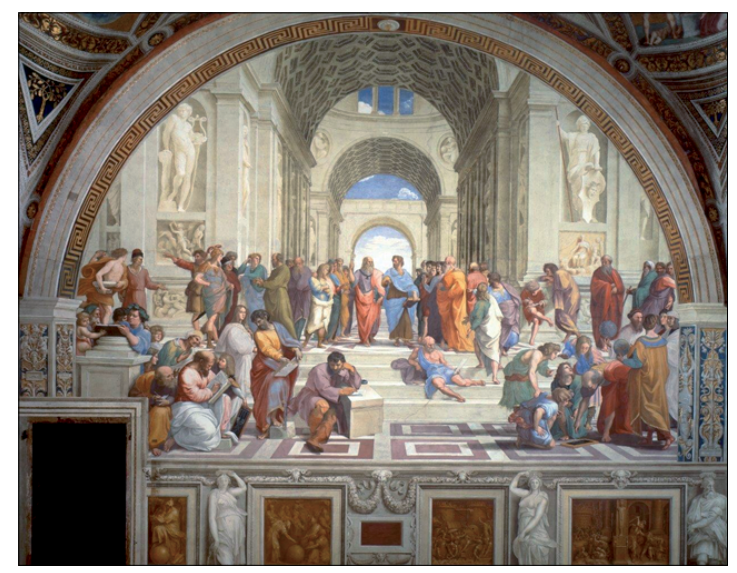


de sus pasadas por otros hospitales (definitivamente hoy flamante maestro de la anestesiología chilena).

Es así que hago la analogía con el famoso fresco de Rafael, pintado a comienzos del siglo XVI y plagado de personajes importantes, en el que me encontraba inmerso y donde si no aprendía era sólo responsabilidad mía. El cuadro decora las habitaciones del Vaticano que hoy en día son conocidas como las estancias de Rafael. Debido a que estaría ubicado sobre la sección de filosofía del papa Julio II, La Escuela de Atenas muestra a los filósofos, científicos y matemáticos más importantes de la época clásica. Con cierta imaginación, hurgando en los rostros de los personajes trato de encontrar en ellos, así como Heráclito tenía los rasgos del propio Miguel Ángel, caras parecidas a Platón, Aristóteles, Sócrates, Averroes, Pitágoras, Arquímedes etc., y relacionarlas con aquellos maestros que circulaban a mi alrededor. Sólo faltaba una Hipatia en ese Servicio, tan misógino en aquéllos tiempos.

Con Samuel, Jorge y Pepe hicimos un muy buen grupo de trabajo, y no sólo trabajábamos bien, sino lo pasábamos bien. El destino o tal vez alguien más concreto, quiso que no pudiera continuar en ese grupo. Es lo que más eché de menos. Lo más probable es que si hubiera seguido allí no estaría recibiendo esta distinción: hubiera sido uno más de ese selecto grupo y me hubiera diluido entre tanta lumbrera de maestros pasados y otros cuantos que quedan por venir. Pero créanme que hubiera valido la pena.

\section{Mi llegada a la Posta Central}

Como ocurría en aquéllos tiempos, y la vida me ha enseñado que en todos los tiempos ocurre así, después de 5 años de trabajar casi en exclusividad en lo que llamé La Escuela de Atenas, tuve que aterrizar a la cruel realidad de encontrar empleo. Fui tratado de retener hasta estirar la cuerda lo máximo posible por la mayoría de los miembros del Servicio, pero ganaron los poderes fácticos y la mentira. El hecho mismo me dolió, pero el apoyo de mis amigos y el saber que tenía muchas alternativas por delante no afectaron $\mathrm{mi}$ decisión de entrar al campo de la salud pública (ahora se usa mucho lo del servicio público, que de servicio va teniendo cada día menos). Una lla- mada de Samuel Torregrosa a uno de sus amigos sirvió de contacto. Max Arriagada me recibió en la Posta Central, a la que seguirán llamando así aunque se hubiera llamado Asistencia Pública, Asistencia Pública Dr. Alejandro del Río o, finalmente, Hospital de Urgencia Asistencia Pública, o sencillamente HUAP (las iniciales y los números nos encantan en estos tiempos). Un jefe acogedor, conocido formador de alumnos de anestesia que se iniciaban en la especialidad antes de recibirse, pero que nos dejó prematuramente: llegué en mayo y falleció en septiembre. Fundador de la UTI del Hospital José Joaquín Aguirre y del Servicio de Anestesiología de la Posta Central. Él hubiera sido un gran candidato para estar en este sitio que yo inmerecidamente estoy ocupando. Le faltó solo llegar a la edad exigida por los estatutos.

Le tomé cariño a la Institución inmediatamente y pasé luego por varias jefaturas con las que tuve buenas y no tan buenas relaciones, según el período. Es bueno que las jefaturas cambien y en nuestro servicio hemos logrado hacerlo sin grandes traumas y en forma bastante democrática. Hoy tenemos una jefa implacable, como está de moda en esta guerra de géneros que han ganado sistemáticamente las mujeres, pero que les ha costado siglos. Aunque algunas veces ha hecho ver que yo soy una especie de "jefe espiritual" (por los años y espero que también por el amplio sentido de la palabra espíritu), la Dra. Clara Luxoro está ahí porque en la práctica ejercía el cargo desde antes: tuvo los merecimientos, la capacidad administrativa y las cualidades ejecutivas que se le conocen. En Max Arriagada y Clara Luxoro simbolizo más de 30 años de estadía en la Institución. No quiero nombrarlos a todos, pero quiero decirles que sinceramente me he sentido siempre querido y respetado por todos. Tengo que hacer una excepción con Mauricio Ramos, que probablemente es quien más recibió mi influencia, la mejor y la peor, y ahora me enorgullece como jefe de un importante servicio público.

Pero cuando yo llegué, los verdaderos jefes eran los jefes de turno y los verdaderos servicios eran los turnos. Una familia que estaba junta varios días y una noche en la semana, con la que se convivía, se bromeaba y se peleaba. Destaco a los Dres. Luis Gauthier y Enrique Ceroni: un maestro y un amigo en cada uno de ellos. Los 
años fueron haciendo de los turnos una olla de grillos y no una familia: "cambia, todo cambia".

Muchos años después, llegué al Servicio de Anestesia de Clínica Dávila, estimulado más que nada por hacer cosas diferentes que por necesidades materiales, ya que con los años estas se van solucionando o van disminuyendo. En los últimos 3 años, que han pasado volando, me encontré con un grupo afiatado, solidario, con un espíritu de equipo y una capacidad de trabajo similar a cualquier hospital público. Aunque mi integración no puede ser la misma, me he ido sintiendo cada vez más como en mi casa.

\section{Docencia en la Posta Central}

El interés por la docencia indudablemente nación con Max Arriagada, pero por su prematura partida fue reemplazado por otras personas que siguieron con entusiasmo, a veces demasiado, la senda de ese maestro. Me es imposible dejar de mencionar el ímpetu de Carlos Reyes, la dedicación de Víctor Hanna y la eficiencia de Renato Chacón. Sin estas tres personas, ninguna de las dos etapas de la docencia en la Posta hubiera sido posible: la primera etapa la constituyó el Programa Ministerial para la Formación de Anestesiólogos y, la segunda, el Programa de Anestesiología dependiente de la Escuela de Postgrado de la Universidad de Santiago. En ninguna de las dos fui protagonista, por lo que sigo preguntándome: ¿Qué hago acá?

La causa de mi reticencia probablemente fue mi manera de hacer las cosas en forma políticamente correcta, deteniéndome en aspectos que posteriormente pudieran ser considerados detalles, pero que en ese momento para mi eran importantes. Estuve a cargo del Programa Ministerial un tiempo, hasta que una situación específica me hizo renunciar; del Programa de la Universidad de Santiago me marginé. Posteriormente, cuando la situación mejoró, me acredité académicamente y he participado activamente en la enseñanza tutorial en el pabellón, que es el lugar donde se aprende anestesia. Uno de los maestros que he mencionado y a quién protegeré su identidad decía: "así como el sexo no se aprende en el Play-Boy, la anestesia no se aprende en los libros, se aprende en el pabellón".

No voy a argumentar mis razones de enton- ces, que las tenía, pero el resultado no me dio la razón. El Programa Ministerial formó a más de 100 especialistas, entre ellos los personajes más destacados de mi Servicio y una gran cantidad de anestesiólogos distribuidos por todo Chile, quiénes obtuvieron además los mejores rendimientos en los exámenes de CONACEM, cuando se les permitió hacerlo. Por otra parte, el Programa de la Universidad de Santiago, después de algunos años de incertidumbre, fue reconocido por API$\mathrm{CE}$; entre ambos programas han contribuido a la formación de 151 anestesiólogos ya egresados y 23 actualmente en formación y ha aportado especialistas a hospitales regionales y a hospitales que son actualmente campos clínicos de la propia Universidad.

Quiero personalizar este logro en algunos de nuestros ex becados, que llevan años prestando sus servicios en hospitales de esta Región: Daniel Villalobos, Héctor Ureta y Cristián Vera, del Plan Ministerial y, Omar Herrera, Sandra Sommer y Carlos López del Programa de la Universidad de Santiago.

\section{Algunas ideas}

Alcanzar este peldaño en mi carrera me motiva a entregar algunas ideas a nuestra comunidad, al Directorio que iniciará su período y a los que vendrán, que, al igual que yo, buscan el engrandecimiento, reconocimiento y prestigio de nuestra querida especialidad. Las planteo como una suerte de desafío para las generaciones actuales y futuras:

- La historia ha demostrado que este galardón hace desaparecer de las pistas a los quienes lo reciben. La ley de la vida es que nos vayamos, pero como nadie tiene el poder de decidir sobre el momento de la partida, no puedo entender que muchos maestros de la anestesia chilena hayan partido sin aportar su experiencia los últimos años. ¿Cuánto hubiera aportado la experiencia de Jorge Urzúa desde 1996, de Héctor Lacassie y Samuel Torregrosa desde 2010, Lucía Volosky y José de la Fuente desde el año pasado? Probablemente unos más y otros menos, pero todos tienen mucho que aportar. Jorge y yo no tenemos intenciones de morirnos todavía y también estamos en condiciones de aportar. Propongo la crea- 
ción de una especie de Senado consultivo que asesore al Directorio en los temas que se le encomienden, cualesquiera que sean, de interés para el mejoramiento de nuestra especialidad: creación de tribunales de ética, cambios de estatutos, etc..

- La Revista Chilena de Anestesia va a pasar a su formato digital definitivo. Mucho he escrito de esto y no voy a agregar más al respecto. Sin embargo, sigo pensando que la SACH no puede dejar completamente de tener un órgano escrito oficial. Podría ser un libro anual sobre un tema específico, que colabore con la educación continua de los especialistas y de los médicos en formación. Una especie de J.E.P.U. para los que conocen el sistema francés. $\mathrm{O}$ una selección de los mejores artículos del año de la Revista. Si el problema es el financiamiento, que se venda, no sólo a los socios. La tecnología permite ahora la impresión de pocos ejemplares a un precio razonable.

- La unificación de los planes de estudio teórico de los diferentes programas de formación, dirigidos por la SACH, sería un gran aporte al mejoramiento de los programas más débiles, para los que al parecer ya no hay vuelta atrás. Esto hiere susceptibilidades, pero, si pudo ser realidad en otras especialidades ¿Porqué no en la nuestra? ¿No es absurdo que se esté impartiendo clases del mismo tema a pequeños grupos, por diferentes docentes, desperdiciando recursos humanos tan necesarios? ¿No sabemos todos quiénes son los mejores en cada tema para organizar diferentes módulos o para hacer cada clase específica? ¿No estaremos sobrecargando a los anestesiólogos en formación con clases teóricas a causa de falta de campos clínicos donde se debe hacer la verdadera docencia: en pabellón? ¿No sería bueno contar con un gran centro de simulación que sirviera para enseñar y evaluar a todos los anestesiólogos, becados y aquellos miembros de la comunidad médica o paramédica que lo requirieran?

- La creación de una distinción intermedia al de Maestro de la Anestesiología Chilena, cuyo solo título es conminatorio. En algunos países, como Canadá, existe el nombramiento del Anestesiólogo del Año, que se da a aquel profesional que se ha destacado por alguna

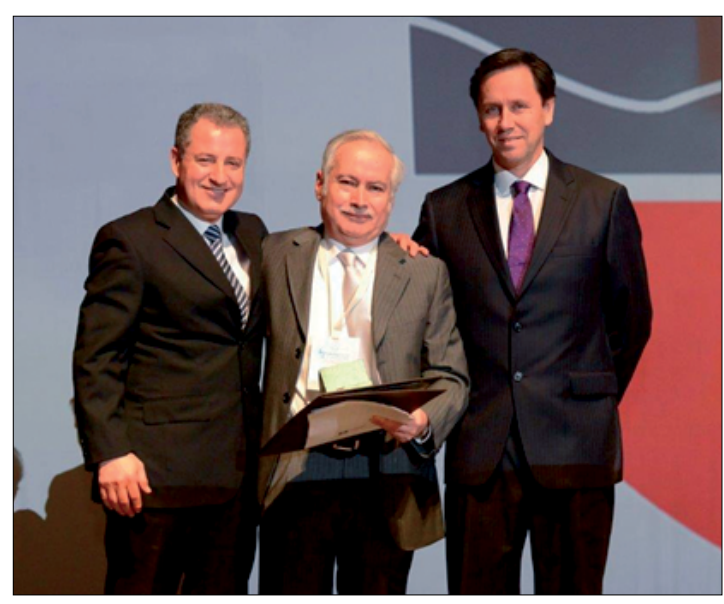

actividad durante un año calendario, sin importar su edad, su grado académico ni su currículo completo.

- He escuchado a algunos colegas conversar sobre la posibilidad de que los cargos del Directorio de la Sociedad y Editor de la Revista deberían ser remunerados. Nada me parecería peor idea. Sólo la dedicación de personas sin intereses ha logrado hacer las cosas bien. No las echemos a perder como ha ocurrido en otros ámbitos del quehacer nacional. Tal vez tendríamos más voluntarios, pero con muchas menos convicciones. Mi padre fue durante muchos años presidente del Club Deportivo de la Universidad de Chile: nunca ganó ni un peso; nunca se robó ni un peso.

\section{Agradecimientos}

Finalmente, aunque espero que el listado no sea como el de la presentación de los Oscar, me es imprescindible hacer algunos reconocimientos a ciertas personas que han sido imprescindibles en mi vida.

A mi, mi mujer y mis hijos, que han sido la razón de mi vida, les agradezco la paciencia por tanto tiempo ocupado en cosas que pudieron haber sido secundarias para otros pero no para mí. Gracias Marisol por el apoyo permanente. Gracias Alejandra por estar. Gracias Felipe porque hubieras querido estar. Y parafraseando a Neruda con su ma-madre, gracias hermano-padre, siempre mi ejemplo a seguir.

Gracias a los miembros del Directorio, que 
tuvieron la osadía de darme su confianza. Tengo allí muchos amigos y como les dije hace un tiempo, siempre me quedará la duda que probablemente la presencia de algunos de Uds. en el actual Directorio, que han tenido una importante participación en mi vida y, otros con los cuales he mantenido una permanente cordial relación, haya precipitado este injustificado nombramiento que, como ya lo he expresado, me llena de orgullo. Se agradece y se disfruta el momento. El tiempo y el chaqueteo habitual de nuestra pequeña comunidad dirá si tuvieron o no razón.

Finalmente, a las nuevas generaciones les digo que si yo estoy recibiendo este reconocimiento, cualquiera de Uds. puede hacerlo. No ha sido un gran esfuerzo. Algún sacrificio, estudio para actualización permanente pero no para confundirse, mucha literatura (discriminando), mucha música (no discriminando: desde el rock ochentero hasta la ópera) y... esperar los 65. Los mexicas decían que sólo por medio del arte, pueden los seres humanos aproximarse a la realidad. ¡Qué gente más sabia habitaba mesoamérica antes de que "nos civilizaran" los europeos!.

Dr. Ricardo Bustamante Bozzo 